\title{
Microstructural Simulation of Three-Point Bending Test with Mo-Si-B Alloy at High Temperature: Sources of Strain Field Localization*
}

\author{
Nuwong CHOLLACOOP**, Amruthavalli P. ALUR*** \\ and K.Sharvan KUMAR*** \\ ** National Metals and Materials Technology Center (MTEC) \\ 114 Thailand Science Park, Pathumthani 12120, THAILAND \\ E-mail: nuwongc@mtec.or.th \\ *** Division of Engineering, Brown University \\ Providence, RI 02912, USA
}

\begin{abstract}
Deformation behavior in three-point bending test of Mo-Si-B alloy was investigated by recourse to finite element analysis (FEA) with microstructure incorporated. This Mo-Si-B alloy consists of hard, brittle $\mathrm{T} 2\left(\mathrm{Mo}_{5} \mathrm{SiB}_{2}\right)$ phase embedded in soft matrix of Mo solid solution. The sample contains pre-crack configuration at the middle in order to study the effect of the second phase (T2 particles) onto a crack tip during the bending test. Various optical micrographs were scanned, digitized and meshed for FEA. It was found that strain localization from the second phase at the crack tip was interfered with that from the loading pin in three-point bending test. Such interference could be reduced by replacement with end moment loading, in order to identify sole strain localization effect from the second phase at the crack tip.
\end{abstract}

Key words: Finite Element Analysis, Three-Point Bending, Mo-Si-B Alloy, High Temperature

\section{Introduction}

In a quest for a new alloy for better high temperature application, especially in the aerospace industry, the Ni-based superalloys, multiphase $\mathrm{Nb}$ - and Mo-based alloys have been targeted for extensive research and development around the world. The complete overviews for both alloys are described elsewhere (1-2). Many attempts have been devoted to characterize its high-temperature properties, e.g. strength, toughness and fatigue. Since experimental observation of high temperature test could sometimes be tedious and painstaking or even impossible to monitor real-time, computational speculation based on experimental data can provide useful insight and predicative ability for property design. The present study was motivated by the experimental observation (3) of recrystallization found during the monotonic and cyclic loading at elevated temperature. Since the recrystallization phenomenon requires strain localization, it is the focus of the present computational study to characterize the degree of localization in this two-phase microstructure. 


\section{Computational Approach}

Figure 1 shows the typical microstructure of Mo-Si-B alloy consisting of a hard, brittle phase $\left(\mathrm{Mo}_{5} \mathrm{SiB}_{2}\right.$ or $\mathrm{T} 2$ shown as black islands in Fig. 1(a)) embedded in a soft matrix of Mo solid solution. For further computational calculation, the micrograph must be processed by adjusting the contrast/brightness of these two phases prior to image analysis for phase identification. Figure 1(b) shows the processed image with clear contrast/brightness threshold between the two phases.

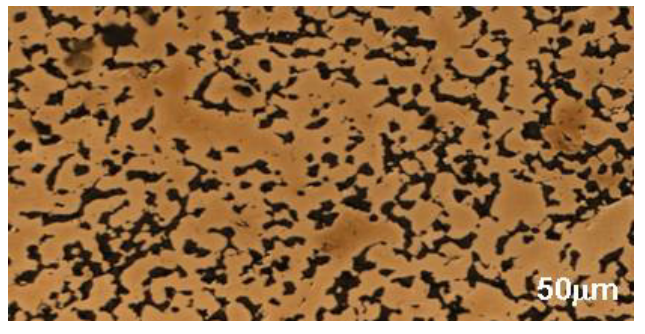

(a)

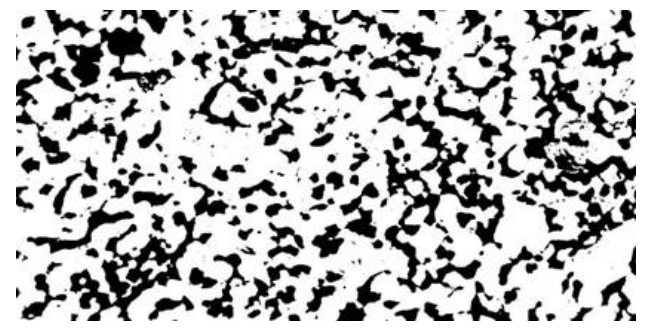

(b)

Figure 1. (a) Typical microstructure of Mo-Si-B alloy with black island of $\mathrm{Mo}_{5} \mathrm{SiB}_{2}$ or $\mathrm{T} 2$ phase embedded in Mo solid solution matrix, and (b) its processed image for simulation.

Various optical micrographs of Mo-6Si-8B (at\%) alloy were optically adjusted to clearly illustrate the size, shape, distribution and spacing characteristics of T2 particles embedded in the Mo solid solution matrix, see example in Fig. 1b. Each processed micrograph was then digitized by image analysis software into an array of 0 or 1 for black or white pixel mapped over the coordinate of the micrograph. Then, this array was passed onto the finite element analysis code for further simulation.

Figure 2(a) depicts the experimental configuration with the sample length of $27 \mathrm{~mm}$, height of $6 \mathrm{~mm}$ and thickness of $3 \mathrm{~mm}$. The sample is sharply notched and compression-compression fatigue pre-cracked for the total pre-crack length of $1.7 \mathrm{~mm}$ (measured under optical microscope) at the center. The geometry of the notch and the minimum fatigue pre-crack length were conformed to ASTM E1820-01. The three-point bending test was set up on the universal testing machine in a vacuum at below $1.33 \times 10^{-4} \mathrm{~Pa}$ with the crosshead displacements of $10^{-3}$ and $10^{-5} \mathrm{~mm} / \mathrm{s}$

For the three-point bending simulation with the test configuration shown in Fig. 2(a), only half of the sample was modeled with the appropriate boundary condition and proper scaling with dimensional consistency (see Fig. 2b). Commercially available FEA package (ABAQUS) was used to simulate three-point bending by quasi-static displacement control of the top pin while holding the bottom pin fixed. The top and bottom pins were approximated as rigid surfaces with frictionless interactions. Along the symmetrical line, the fatigue pre-crack was modeled as mathematical sharp allowing only vertical degree of freedom on the uncrack portion and unconstrained deformation on the crack portion. No crack propagation was allowed in the present model. Since the sample thickness is half the sample height $(3 \mathrm{~mm})$, plane strain element was used.

Both phases were assigned constitutive relations obtained from experimental observations at $1000 \mathrm{~K}$ (4). In other words, the hard T2 phase was treated as being elastic with Young's modulus of $383 \mathrm{GPa}$ and Poisson's ratio of 0.26 (5); whereas, the Mo matrix was treated as elastic-plastic with Young's modulus of $324 \mathrm{GPa}$, Poisson's ratio of 0.29 , yield strength of $400 \mathrm{MPa}$ and linear work-hardening rate of $200 \mathrm{MPa}$ per unit strain (6). Five micrographs were randomly taken and analyzed for the volume fraction. The one, which has the closet volume fraction to the average value from five micrographs, was chosen as the representative microstructure. Figures $2 \mathrm{c}$ and $2 \mathrm{~d}$ show the original 
microstructure of $180 \times 404 \mu \mathrm{m}^{2}$ and its digitization into FEA model with uniform element size of $2 \times 2 \mu \mathrm{m}^{2}$ (a total of 18,180 elements), respectively. The mesh resolution around the crack tip is shown in Figure 2e (only meshes for T2 phase are shown).

(a)

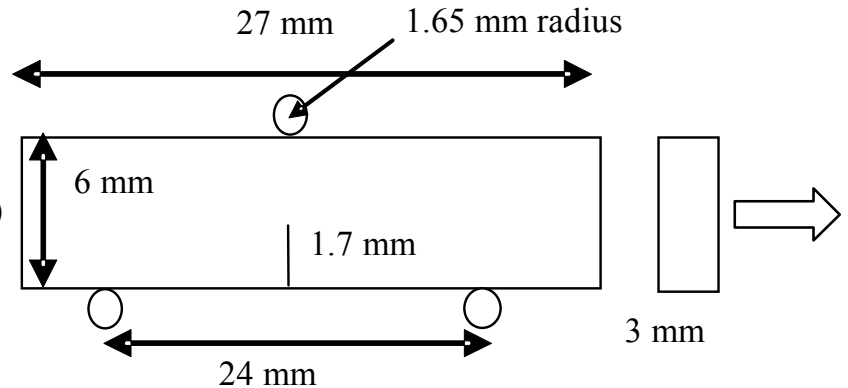

$.65 \mathrm{~mm}$ radius (b)

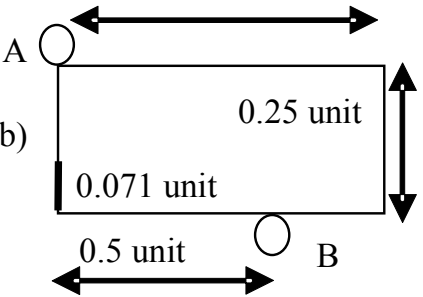

(c)

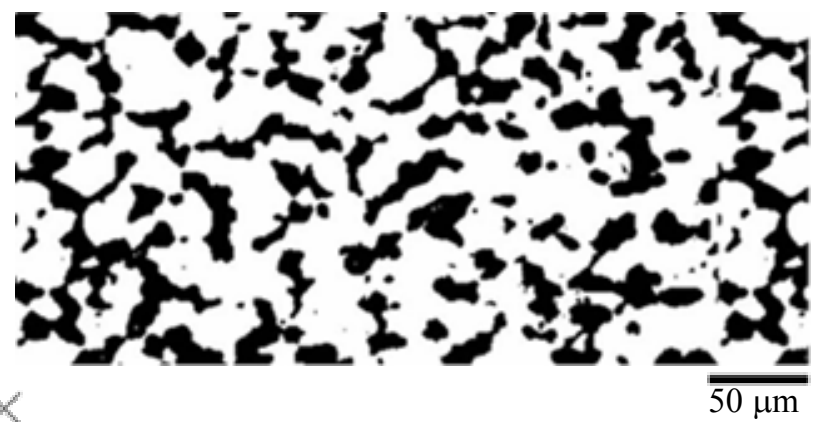

Top pin

(d)

(e)

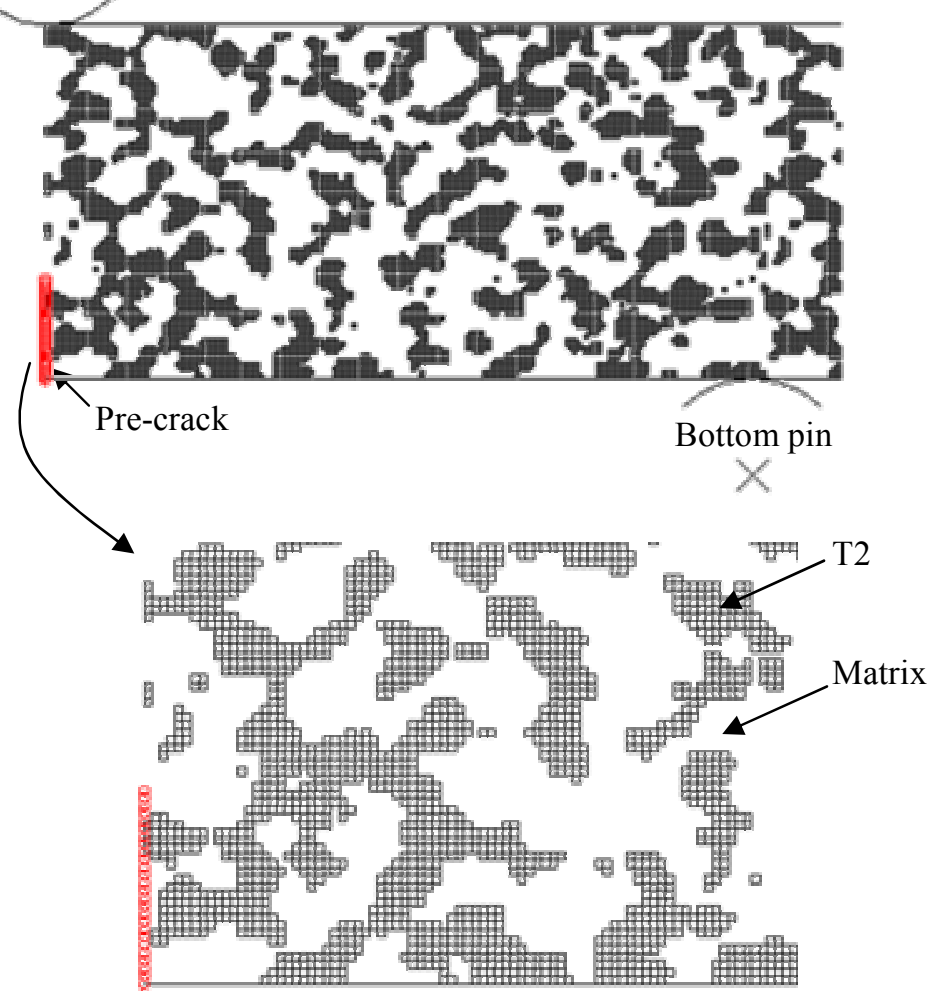

Figure 2. (a) Experimental configuration of the three-point bending test with (b) the half cell model for simulation due to symmetry, (c) the original microstructure with (d) its translation into FEA model with top and bottom pins, and (e) the mesh resolution around the crack tip (only meshes for $\mathrm{T} 2$ phase are shown) 


\section{Results \& Discussion}

The contour plots of Mises stress (Fig. 3a-c) and plastic equivalent strain PEEQ (Fig. 3d-f) were extracted from the simulation results at various vertical displacements of the top pin (U2). Since hard T2 phase can only deform elastically while soft Mo phase can accommodate plasticity, the high Mises stress is observed in the T2 phase ahead of the crack tip even at early U2 displacement. Similar regions are also visible due to the indentation effect by the loading top pin. With larger U2 displacement, the stress localization expands further on the network of $\mathrm{T} 2$ clusters around the crack tip, interacting with the stress localization from the indentation of the top pin. Then, it is difficult to isolate the individual effect. If the brittle failure criterion is provided for the computation, the T2 phase may crack to relieve these stresses.

On the other hand, small extent of plastic deformation is observed around the crack tip at early U2 displacement (see Fig. 3d). With larger U2 displacement, the strain localization is still focused at the crack tip with additional circular ring pattern (indicated by arrows in Fig. 3f) away from the crack tip. The interaction from the indentation loading pin starts to emerge out also. Previous study (3) showed that this plastic localization is treated as an indicator for local dislocation density with necessary strain energy to drive recrystallization found in the interrupted fatigue tests. Note that the particle/matrix interfaces readily provide easy nucleation sites. As expected, both stress and strain localizations do not appear for homogeneous material, as shown in Fig. 3g, when both $\mathrm{T} 2$ and Mo matrix were assigned same constitutive relation.

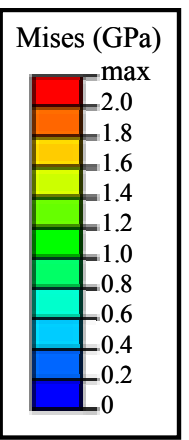

(a)

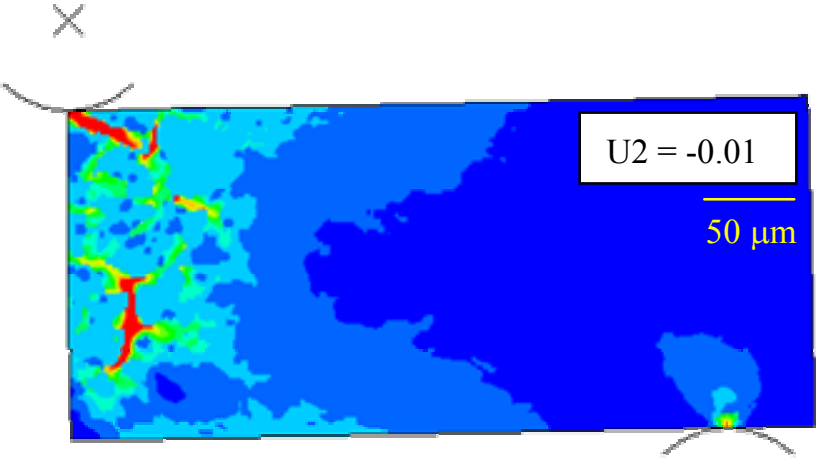

(b)
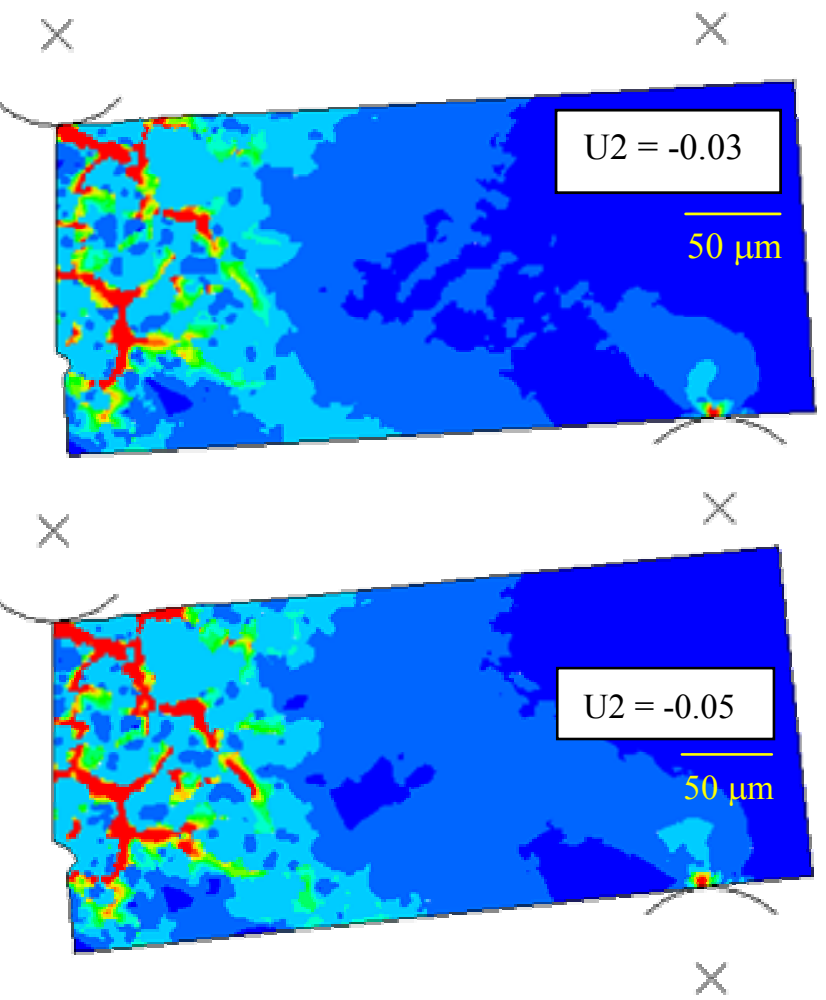


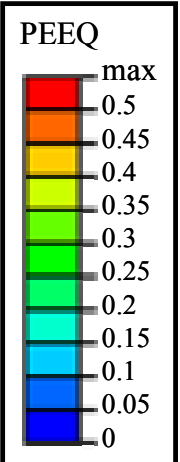

(d)

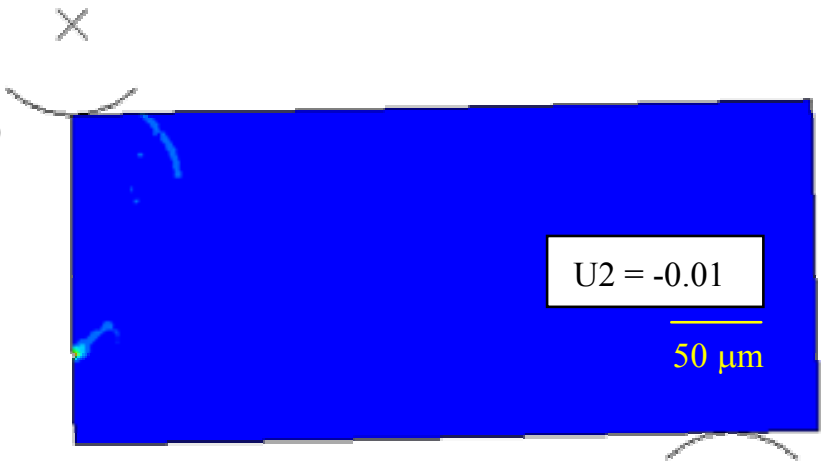

(e)

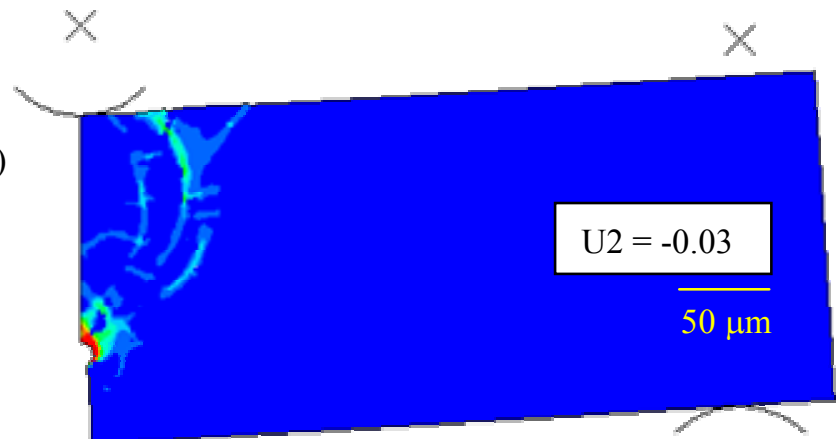

(f)

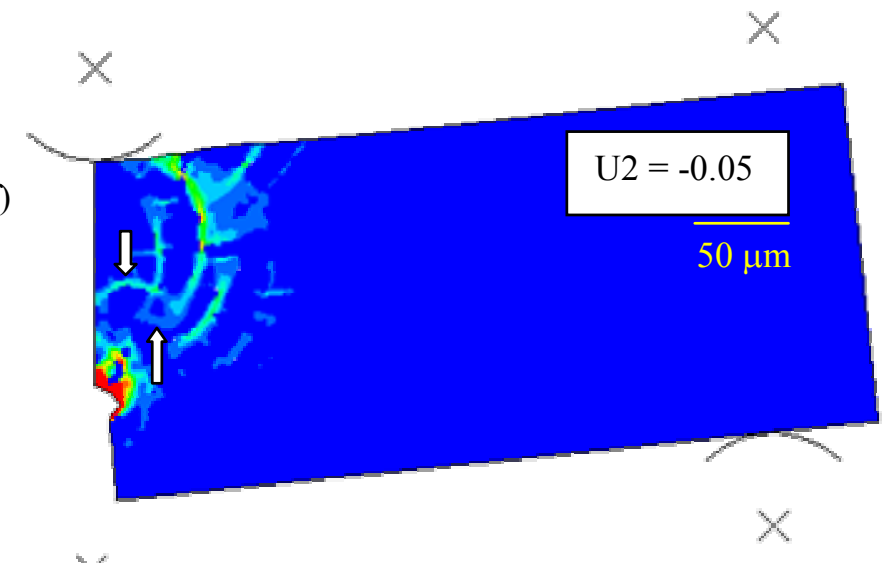

(g)

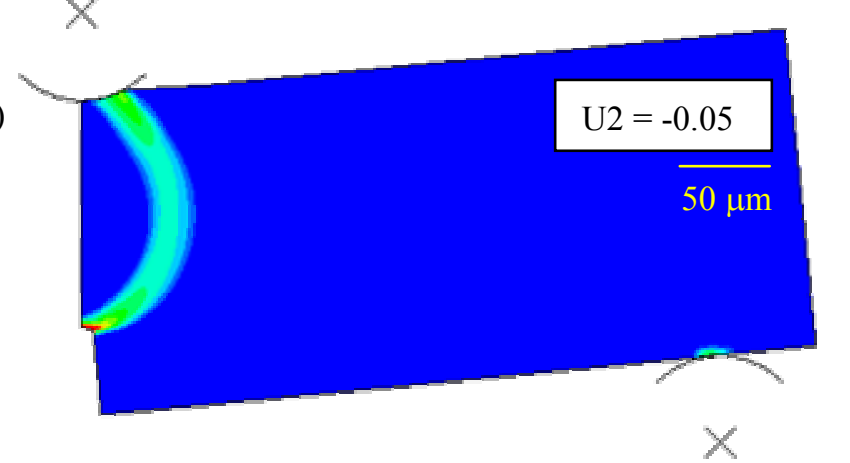

Figure 3. Mises stress contour plot at U2 of (a) -0.01 , (b) -0.03 , (c) -0.05 ; and PEEQ contour plot at $\mathrm{U} 2$ of (d) -0.01 , (e) -0.03 , (f) -0.05 and (g) -0.05 if both phases were assigned the same constitutive relation (homogenous microstructure)

In an attempt to isolate individual localization from the crack tip and the indentation of top pin, the distance between the crack tip and the top pin contact point can be farther separated by using the larger microstructure while maintaining the specimen geometry. 
Figure $4 \mathrm{a}$ shows the digitized new microstructure of $360 \times 808 \mu \mathrm{m}^{2}$ (four-fold to the $180 \mathrm{x} 404$ $\mu \mathrm{m}^{2}$ span in Fig. 2c). Fig. $4 \mathrm{~b}$ shows the contour plot of PEEQ at U2 displacement of -0.035 . It is obvious that the interaction between the crack tip and the indentation of top pin still exists. Another attempt is to replace the pushing top pin by its equivalent end moment. Figure $4 \mathrm{c}$ shows the contour plot of PEEQ at the same U2 as that in Fig. $4 \mathrm{~b}$. Comparison between Figs. $4 \mathrm{~b}$ and $4 \mathrm{c}$ at the same U2 suggests that the interaction between the strain field from crack tip and the strain field from indentation of the top pin contact can be reduced via end moment loading.

(a)
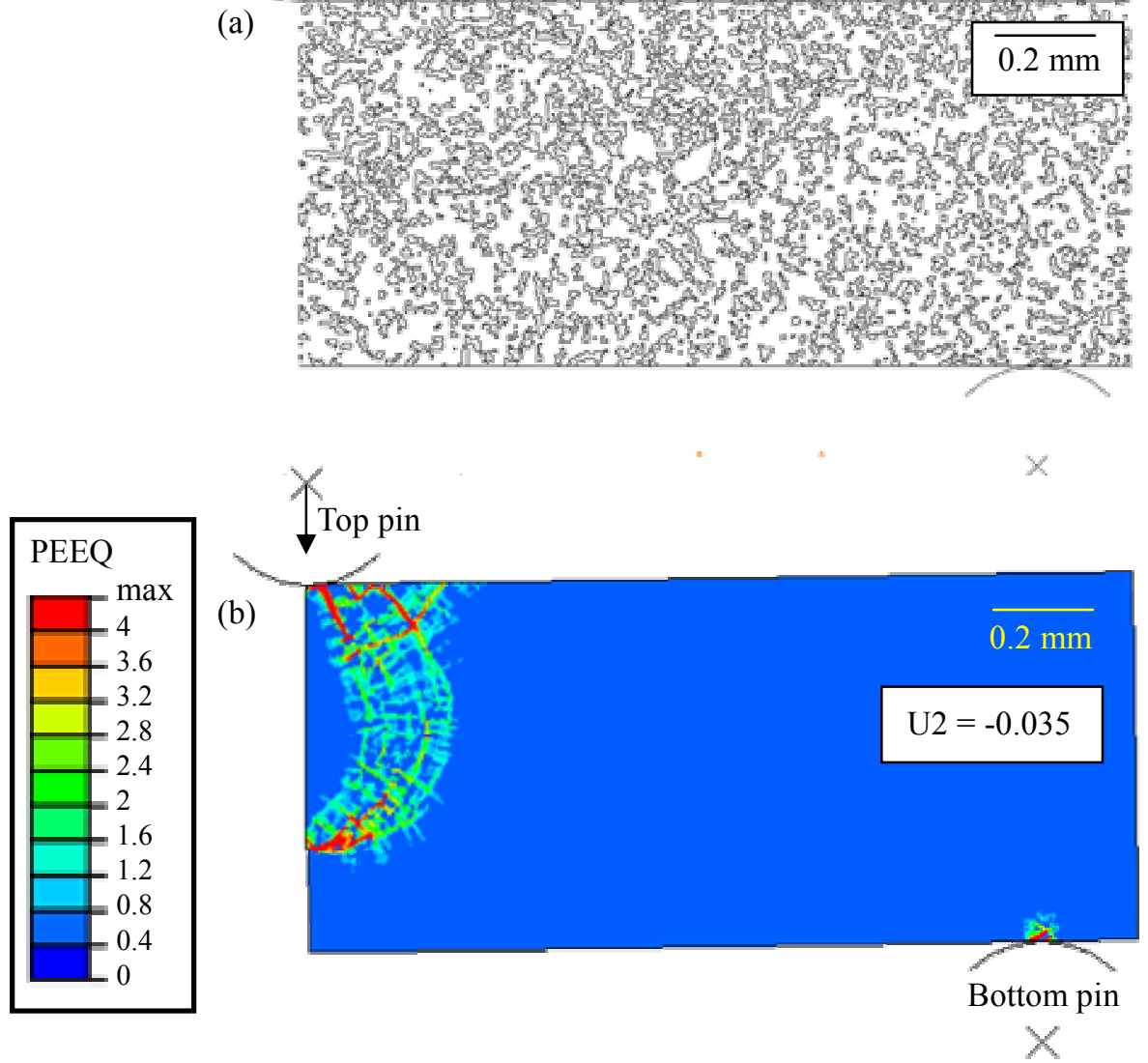

(c)

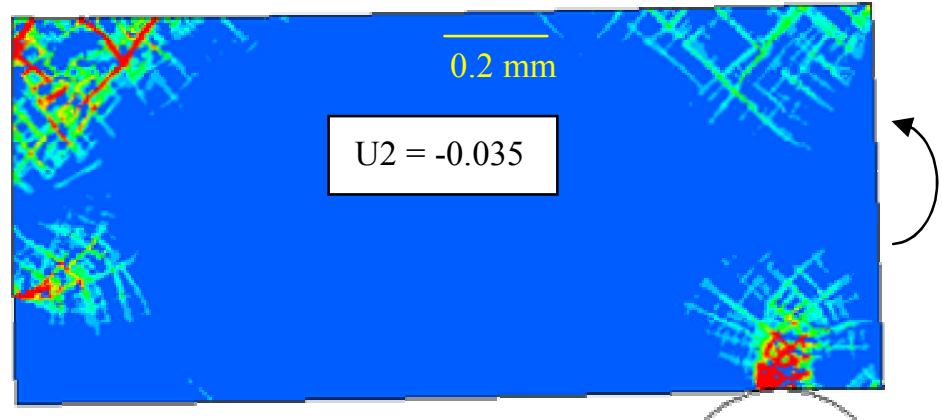

$\times$

Figure 4. (a) Four-fold microstructure to that in Fig. 2(c) with PEEQ contour plot at the vertical displacement $\mathrm{U} 2=-0.035$ by (b) indentation and (c) bending moment. 


\section{Conclusion}

Computation modeling of three-point bending of heterogeneous microstructure consisting of hard T2 particles embedded in soft Mo matrix was conducted. Stress and strain localizations were investigated based on the differences in the constitutive relations of the two phases. This strain localization ahead of the crack tip, which was considered as a driving force for recrystallization in the vicinity of the growing crack, was investigated on the basis of $\mathrm{T} 2$ particles and the loading condition. Interactions of strain fields from crack tip and top pin indentation prevent clear interpretation of individual effect. These interactions are reduced when the top pin loading is replaced by the end moment loading while increasing the distance between crack tip and top pin contact does not reduce such interactions. Thus, careful interpretation of the strain localization from second phase (T2) particles ahead of the crack tip is warranted, with the identification of the strain localization from the loading configuration.

\section{References}

(1) Bewlay, B.P. et al, Ultrahigh-Temperature Nb-Silicide-Based Composites, MRS Bulletin, Vol. 28, (2003), pp. 646-653.

(2) Dimiduk, D.M. and Perepezko, J.H., Mo-Si-B Alloys: Developing a Revolutionary Turbine-Engine Material, MRS Bulletin, Vol. 28, (2003), pp. 639-645.

(3) Alur, A.P. et al, Creep/Environment Effects on Crack Growth in a Mo-Si-B Alloy, Acta Mater., Vol. 55, (2007), pp. 961-974.

(4) Alur, A.P. et al, High-temperature compression behavior of Mo-Si-B alloys, Acta. Mater., Vol. 52, (2004), pp 5571-5587.

(5) Ito K. et al, Physical and mechanical properties of single crystals of the T2 phase in Mo-Si-B system, Intermetallics, Vol. 6, (2001), pp 591-602

(6) Cheng J. et al, A unified constitutive model for strain-rate and temperature dependent behavior of molybdenum, Mechanics of Materials, Vol. 33, (2001), pp 603-616 